\title{
Synchronization and Frustration in Coupled Large-scale Polygonal Oscillatory Networks
}

\author{
Yoko Uwate \\ Dept. of Electrical and Electronic Engineering, \\ Tokushima University \\ 2-1 Minami-Josanjima, Tokushima, Japan \\ Email: uwate@ee.tokushima-u.ac.jp
}

\author{
Yoshifumi Nishio \\ Dept. of Electrical and Electronic Engineering, \\ Tokushima University \\ 2-1 Minami-Josanjima, Tokushima, Japan \\ Email: nishio@ee.tokushima-u.ac.jp
}

\begin{abstract}
In this study, synchronization phenomena observed in coupled polygonal oscillatory networks with frustration is investigated. In this oscillatory system, dilemma is created by the shared branch. We investigate the phase difference between adjacent oscillators when the number of oscillators connected to one side of the polygonal network is increased for considering the large-scale oscillatory networks.
\end{abstract}

\section{INTRODUCTION}

Coupled oscillatory systems are good models to express essential role of high-dimensional nonlinear phenomena occurring in the field of natural sciences. Synchronization phenomena have been extensively reported in physical [1]-[4], biological [5],[6] and electrical [7],[8] systems. Endo et al. have presented the details of a theoretical analysis and corresponding circuit experiments on electrical circuits oscillators arranged in a ladder, a ring and in a two-dimensional array topology [9]-[11]. Moreover, coupled oscillatory systems can also produce interesting phase patterns, including wave propagation, clustering and complex patterns [12]. Therefore, we expect that synchronization phenomena can be applied for modeling of the high-functional information processing in the human brain.

On the other hand, there are several types of polygonal network structures (e.g. Honeycomb structure and crystal structure) in the natural science. Generally, for the studies of large-scale network using coupled oscillators, a ring, a ladder and a two dimensional array structure are often investigated. However, there are not many discussions about coupled polygonal oscillatory networks by using electrical oscillators. Furthermore, it is interesting to investigate the effect of some sort of dilemmas or frustrations for the synchronization of coupled oscillatory systems and important to understand the mechanism of synchronization observed in the field of natural science.

In our previous studies, synchronization phenomena in coupled polygonal oscillatory networks shared with one branch have investigated. Here, the number of oscillators coupled to the one polygonal network is set to odd number. We considered two identical oscillatory networks were coupled by sharing a branch as a symmetrical system. In this oscillatory system, frustration was occurred by the shared branch. We confirmed that the phase difference between the shared oscillators is shifted, then other oscillators synchronize to compensate this phase shift.

In this study, we consider the circuit model which is that odd number of polygonal network and even number polygonal network are coupled by sharing one branch. For considering the large-scale networks, the number of oscillators conncected to the one side of polygonal network is increased untill around 100. First, the phase difference and frustrations are investigated when triangular oscillatory network and the even number polygonal network are coupled by sharing one branch. Next, we consider the quadrangular oscillatory networks and odd number polygonal network are coupled with one branch.

\section{Coupled Triangular Network and Even Number Polygonal NeTWORK}

Figure 1 shows the circuit model which is that triangular network and even number polygonal network are coupled with one branch. Where $N_{e}$ denotes the number of coupled oscillators of even polygonal network. As an example, the coupling method between the adjacent oscillators when coupled triangular network and quadrangular network are coupled is shown in Fig. 2.

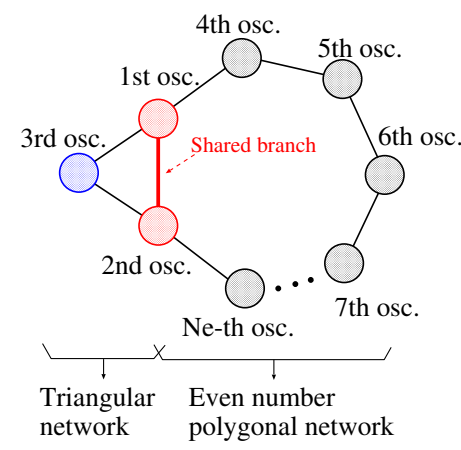

Fig. 1. Conceptual Circuit Model of Coupled Triangular Network and Even Number Polygonal Network $\left(N_{e}=4,6,8,10, \ldots 100\right)$.

The $v_{k}-i_{R k}$ characteristics of the nonlinear resistor are approximated by the following third order polynomial equation,

$$
i_{R k}=-g_{1} v_{k}+g_{3} v_{k}^{3} \quad\left(g_{1}, g_{3}>0\right) .
$$

The normalized circuit equations governing the circuit are expressed as 


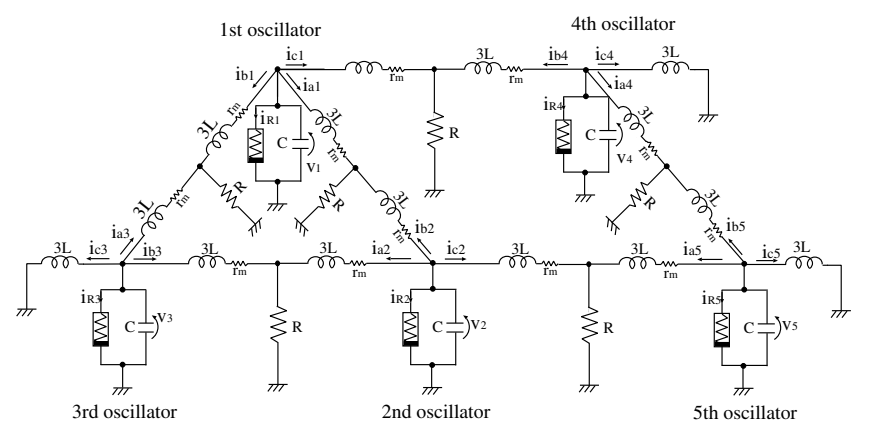

Fig. 2. Circuit Model for Coupling Method (ex. Triangular Network and Quadrangular Network).

[First oscillator]

$$
\left\{\begin{aligned}
\frac{d x_{1}}{d \tau} & =\varepsilon\left(1-\frac{1}{3} x_{1}^{2}\right) x_{1}-\left(y_{a 1}+y_{b 1}+y_{c 1}\right) \\
\frac{d y_{a 1}}{d \tau} & =\frac{1}{3}\left\{x_{1}-\eta y_{a 1}-\gamma\left(y_{a 1}+y_{b 2}\right)\right\} \\
\frac{d y_{b 1}}{d \tau} & =\frac{1}{3}\left\{x_{1}-\eta y_{b 1}-\gamma\left(y_{a 3}+y_{b 1}\right)\right\} \\
\frac{d y_{c 1}}{d \tau} & =\frac{1}{3}\left\{x_{1}-\eta y_{c 1}-\gamma\left(y_{b 3}+y_{c 1}\right)\right\}
\end{aligned}\right.
$$

[Second oscillator]

$$
\left\{\begin{aligned}
\frac{d x_{2}}{d \tau} & =\varepsilon\left(1-\frac{1}{3} x_{2}^{2}\right) x_{2}-\left(y_{a 2}+y_{b 2}+y_{c 2}\right) \\
\frac{d y_{a 2}}{d \tau} & =\frac{1}{3}\left\{x_{2}-\eta y_{a 2}-\gamma\left(y_{a 2}+y_{b 3}\right)\right\} \\
\frac{d y_{b 2}}{d \tau} & =\frac{1}{3}\left\{x_{2}-\eta y_{b 2}-\gamma\left(y_{a 1}+y_{b 2}\right)\right\} \\
\frac{d y_{c 2}}{d \tau} & =\frac{1}{3}\left\{x_{2}-\eta y_{c 2}-\gamma\left(y_{a 5}+y_{c 2}\right)\right\}
\end{aligned}\right.
$$

[Third oscillator]

$$
\left\{\begin{aligned}
\frac{d x_{3}}{d \tau} & =\varepsilon\left(1-\frac{1}{3} x_{3}^{2}\right) x_{3}-\left(y_{a 3}+y_{b 3}+y_{c 3}\right) \\
\frac{d y_{a 3}}{d \tau} & =\frac{1}{3}\left\{x_{3}-\eta y_{a 3}-\gamma\left(y_{a 3}+y_{b 1}\right)\right\} \\
\frac{d y_{b 3}}{d \tau} & =\frac{1}{3}\left\{x_{3}-\eta y_{b 3}-\gamma\left(y_{a 2}+y_{b 3}\right)\right\} \\
\frac{d y_{c 3}}{d \tau} & =\frac{1}{3}\left\{x_{3}-\eta y_{c 3}\right\}
\end{aligned}\right.
$$

[Fourth oscillator]

$$
\left\{\begin{aligned}
\frac{d x_{4}}{d \tau} & =\varepsilon\left(1-\frac{1}{3} x_{4}^{2}\right) x_{4}-\left(y_{a 4}+y_{b 4}+y_{c 4}\right) \\
\frac{d y_{a 4}}{d \tau} & =\frac{1}{3}\left\{x_{4}-\eta y_{a 4}-\gamma\left(y_{a 4}+y_{b 5}\right)\right\} \\
\frac{d y_{b 4}}{d \tau} & =\frac{1}{3}\left\{x_{4}-\eta y_{b 4}-\gamma\left(y_{b 4}+y_{c 1}\right)\right\} \\
\frac{d y_{c 4}}{d \tau} & =\frac{1}{3}\left\{x_{4}-\eta y_{c 4}\right\}
\end{aligned}\right.
$$




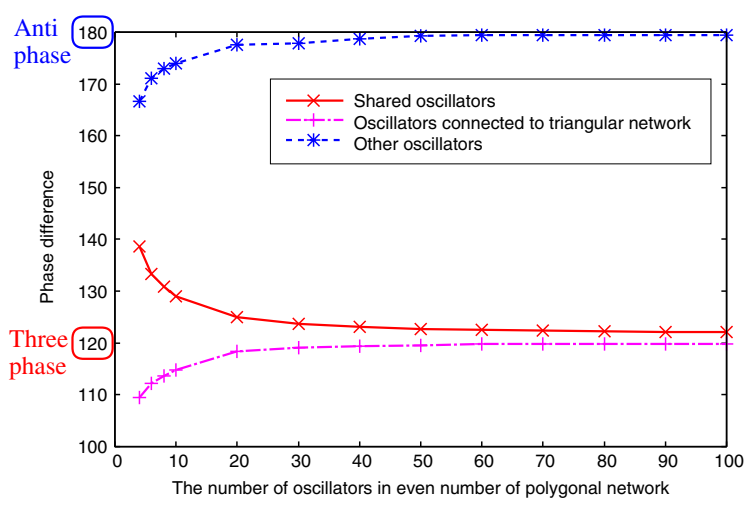

Fig. 3. Phase difference with $N_{e}$.

The equation of the frustration is defined by decrease ratio of the amplitude as follows.

$$
F=\frac{2.0-A_{k}}{2.0} \times 100[\%],
$$

where, $A_{k}$ denotes the amplitude of $k$ th oscillator when the oscillators are coupled. There are three types of frustrations depending on the coupled oscillators as follows.

- Shared oscillators.

- Third oscillator.

- Oscillators connected to the even number polygonal network.

The simulation results of the frustration by increasing $N_{e}$ is shown in Fig. 4. We can see that the third oscillator has most strong frustration in this circuit model of Fig. 1. Furthermore, the frustration of oscillators connected to the even number polygonal network has lower value than the other oscillators and show the most change from 0.7 to 0.2 .

From this simulation result, the frustration decreases by increasing the number of coupled oscillators of even number polygonal network.

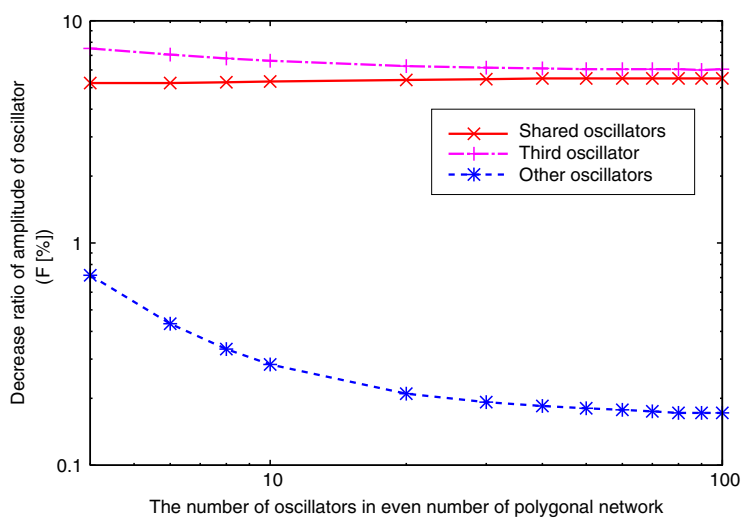

Fig. 4. Decrease ratio of amplitude of oscillators (frustration) with $N_{e}$.

Next, the time wave forms of the voltage of the capacitance in the triangular oscillatory network when the number of even number polygonal network is set to $N_{e}=4,100$ is shown in Fig. 5. We can see that the triangular network tends to the three-phase synchronization by increasing $N_{e}$.

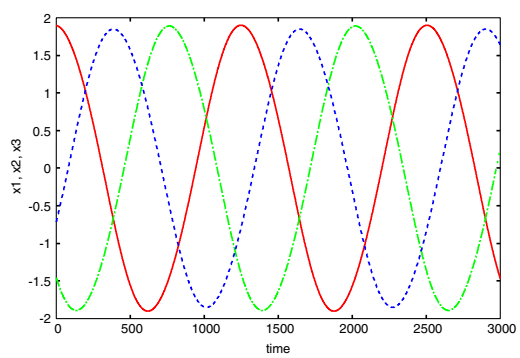

(a) $N_{e}=4$.

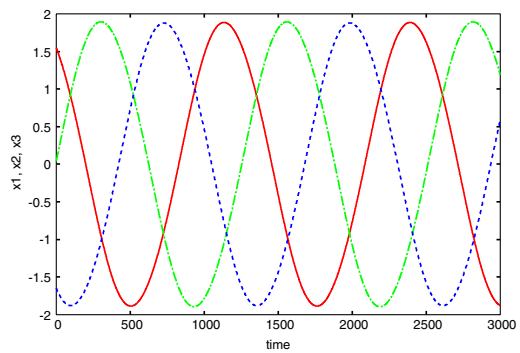

(b) $N_{e}=100$.

Fig. 5. Time wave forms of $x_{1}, x_{2}$ and $x_{3}$.

\section{COUPled QuAdRANGUlar OSCILlatory Network AND OdD Number Polygonal NeTWORK}

In this section, we consider the circuit model which is that quadrangular network and odd number polygonal network are coupled with one branch as shown in Fig. 1 . Where $N_{o}$ denotes the number of coupled oscillators of odd polygonal network. The normalized circuit equations are obtained by same way of above section.

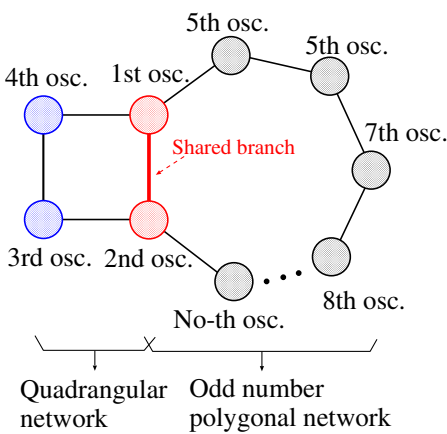

Fig. 6. Conceptual Circuit Model of Coupled Quadrangular Network and Odd Number Polygonal Network $\left(N_{o}=3,5,7,9, \ldots 99\right)$.

\section{A. Phase Difference}

Figure 7 shows the phase differences when $N_{o}$ is changed from 5 to 101 . The three types of the phase differences have the different value when the $N_{o}$ is smaller than 50 . We can see that all types of the phase differences tend to the anti-phase state (phase difference: $180^{\circ}$ ) by increasing $N_{o}$.

\section{B. Frustration}

The simulation results of the frustration by increasing $N_{o}$ is shown in Fig. 8. The frustrations of the quadrangular oscillatory network is smaller than the others. Furthremore, we 


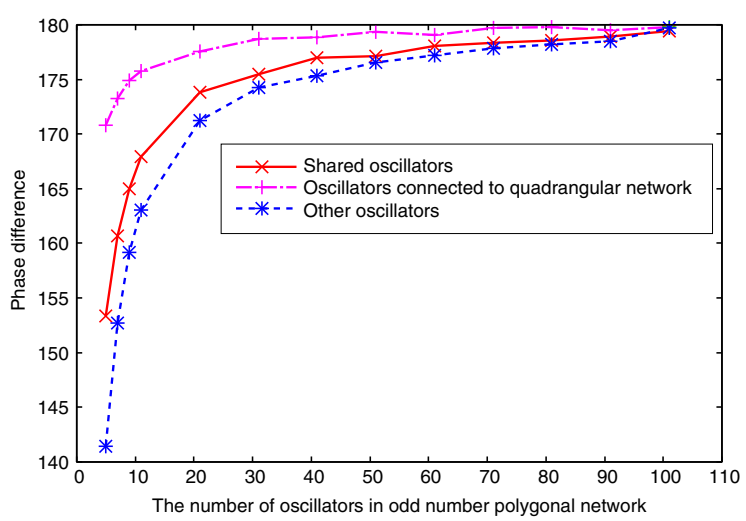

Fig. 7. Phase difference with $N_{o}$.

confirm that the frustrations decrease by increasing the number of coupled oscillators of odd number polygonal network.

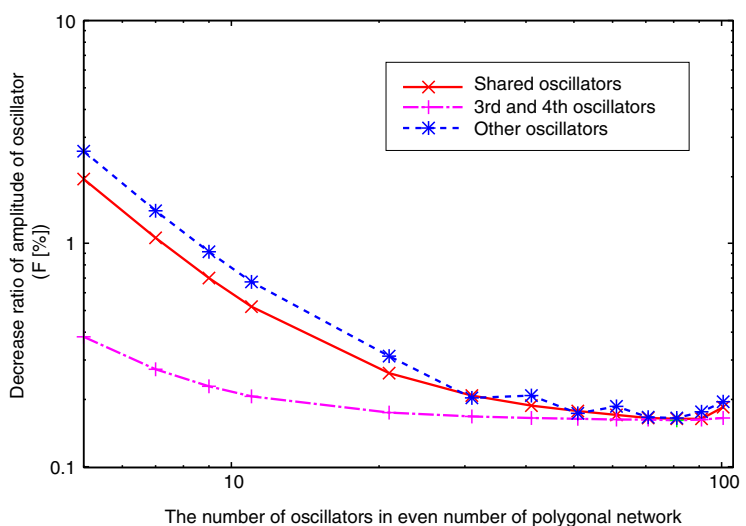

Fig. 8. Decrease ratio of amplitude of oscillators (frastration) with $N_{O}$.

Finally, the time wave forms of the voltage of the capacitance in the triangular oscillatory network when the number of even number polygonal network is set to $N_{o}=5,101$ is shown in Fig. 9. We can see that the quadrangular network closes to the anti-phase synchronization by increasing $N_{o}$.

\section{CONCLUSIONS}

In this study, we have investigate synchronization observed in the circuit model which is that odd number of polygonal network and even number polygonal network are coupled by one branch. First, the phase difference and frustrations were investigated when triangular oscillatory networks is fixed as odd number polygonal network and the number of even number polygonal network is changed from 4 to 100 . By computer simulations, we confirmed that the triangular network closes to the three-phase state and the even number polygonal network closes to the anti-phase states when the circuit system becomes large.

Next, we considered the quadrangular oscillatory networks and odd number polygonal network are coupled with one branch. In this case, we confirmed that all of coupled oscillators tend to the anti-phase state by increasing $N_{o}$.

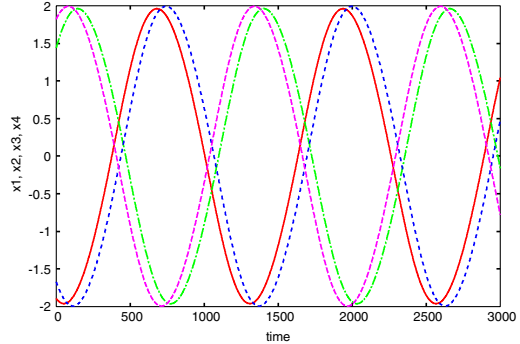

(a) $N_{o}=5$.

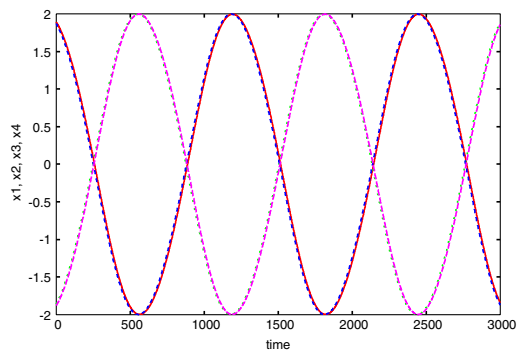

(b) $N_{o}=101$.

Fig. 9. Time wave forms of $x_{1}, x_{2}, x_{3}$ and $x_{4}$.

\section{ACKNOWLEDGMENT}

This work was partly supported by JSPS Grant-in-Aid for Young Scientists 23700269.

\section{REFERENCES}

[1] L.L. Bonilla, C.J. Perez Vicente and R. Spigler, "Time-periodic phases in populations of nonlinearly coupled oscillators with bimodal frequency distributions," Physica D: Nonlinear Phenomena, vol.113, no.1, pp.7997, Feb. 1998.

[2] J.A. Sherratt, "Invading wave fronts and their oscillatory wakes are linked by a modulated traveling phase resetting wave," Physica D: Nonlinear Phenomena, vol.117, no.1-4, pp.145-166, June 1998.

[3] G. Abramson, V.M. Kenkre and A.R. Bishop, "Analytic solutions for nonlinear waves in coupled reacting systems," Physica A: Statistical Mechanics and its Applications, vol.305, no.3-4, pp.427-436, Mar. 2002.

[4] I. Belykh, M. Hasler, M. Lauret and H. Nijmeijer, "Synchronization and graph topology," Int. J. Bifurcation and Chaos, vol.15, no.11, pp.34233433, Nov. 2005

[5] C.M. Gray, "Synchronous oscillations in neural systems: mechanisms and functions," J. Computational Neuroscience, vol.1, pp.11-38, 1994.

[6] R. Stoop and C. Wagner, "Neocortex's architecture optimizes computation, information transfer and synchronizability, at given total connection length," International Journal of Bifurcation and Chaos, vol.17, no.7, pp.2257-2279, 2007.

[7] T. Suezaki and S. Mori, "Mutual synchronization of two oscillators," Trans. IECE, vol.48, no.9, pp.1551-1557, Sep. 1965.

[8] H.B. Fotsina and J. Daafouza, "Adaptive synchronization of uncertain chaotic colpitts oscillators based on parameter identification" Physics Letters A, vol.339, pp.304-315, May. 2005.

[9] T. Endo and S. Mori, "Mode analysis of a multimode ladder oscillator," IEEE Trans. Circuits Syst., vol.23, pp.100-113, Feb. 1976.

[10] T. Endo and S. Mori, "Mode analysis of two-dimensional low-pass multimode oscillator,' IEEE Trans. Circuits Syst., vol.23, pp.517-530, Sep. 1976.

[11] T. Endo and S. Mori, "Mode analysis of a ring of a large number of mutually coupled van der Pol oscillators," IEEE Trans. Circuits Syst., vol.25, no.1, pp.7-18, Jan. 1978.

[12] M. Yamauchi, Y. Nishio and A. Ushida, "Phase-waves in a ladder of oscillators" IEICE Trans. Fundamentals, vol.E86-A, no.4, pp.891-899, Apr. 2003.

[13] Y. Uwate, Y. Nishio and R. Stoop, "Synchronization in Three Coupled van der Pol Oscillators with Different Coupling Strength," Proc. of NCSP'10, pp. 109-112, Mar. 2010. 